\title{
Model roles for role models
}

\author{
The majority of biological research is concentrated on a handful of species for valid practical reasons. \\ But it is important that such pragmatism does not distort our view of life's complexity.
}

Much of modern biology is founded on the concept of the model organism. In essence, it is assumed that by studying a particular organism in sufficient detail the knowledge gained can be carried over to help understand other creatures. All life uses the same basic processes so the individual characteristics of an organism are less important than its similarities to other creatures; or so it is hoped. And yet one of the essential characteristics of life is not its uniformity but its diversity. Indeed, that diversity is usually what first attracts us to the study of the natural world.

The important characteristics of a model organism relate more to its ease of study than whether it is a 'typical' representative of a division of life. A model should be small in size and so easy to maintain in a laboratory environment; it should have a small genome and be amenable to molecular biological manipulation; it should be fast growing and have a short life cycle so that breeding experiments can be performed within the duration of a standard research project. These are all attributes that are shared by classic animal models such as Escherichia coli, Saccharomyces cerevisiae, Caenorhabditis elegans, fruit flies, zebrafish and mice.

By these criteria Arabidopsis thaliana makes an excellent model plant. It is small in stature with a relatively compact genome (at least for a plant) and is so robust that it now grows wild on every continent on the planet with the possible exception of Antarctica. It is currently flowering contentedly in the gaps in the pavement outside the Nature offices, although somewhat overshadowed by its larger cousin shepherd's purse, Capsella bursa-pastoris. But for all its utility Arabidopsis does not have a great deal in common with the cereal grasses that form the staple to most human diets. In this isssue of Nature Plants, Thomas Brutnell discusses efforts to establish a model grass to aid the translation of basic research into agricultural improvements (see Comment by T. Brutnell). Much research is of course performed on the major cereal crops themselves - rice, wheat, maize - but the processes of domestication have produced complicated organisms that are difficult to study. Instead Brutnell champions the use of Brachypodium distachyon and Setaria viridis as simple grasses possessing the qualities

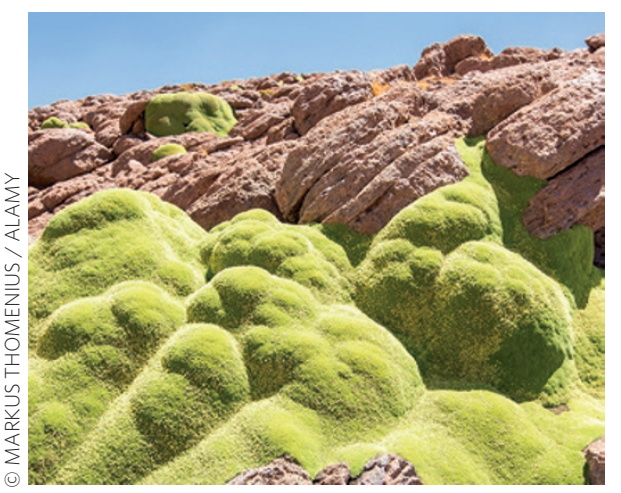

that make good model organisms but whose biology is much closer to that of crop varieties than is Arabidopsis.

Some researchers already consider Arabidopsis as too complex an organism to help inform us about the fundamentals of plant biology and evolution. Plants whose common ancestor with trees and grasses was much more ancient than that of Arabidopsis are becoming popular subjects of investigation. The moss Physcomitrella patens, whose standard laboratory strain was collected from a wood near Cambridge, UK, in 1962, was the subject of over 500 publications in the past five years, while the liverwort Marchantia polymorpha could be the model plant du jour with 126 publications over the same period.

There are also plants whose eccentric and fascinating behaviour have them permanently labelled as 'non-models'. We have examples of these in Nature Plants as well. Christian Schulze Gronover and colleagues report on the enzymes in dandelion, Taraxacum brevicorniculatum, that synthesize natural rubber (see Article by J. Epping et al.). It may come as a surprise that the rubber tree, Hevea brasiliensis, is not the only source of natural rubber. In fact during the Second World War the Soviet Union cultivated dandelions on a large scale as supplies of Hevea rubber from south-east Asia were threatened. There is currently a resurgence of interest in dandelion rubber for medical uses due to fewer allergic reactions, while dandelions could also prove a more sustainable crop.

Orchids are another non-model plant group that nevertheless reward study. Chang-Hsien Yang and his colleagues have looked at the molecular basis behind the creation of their beautifully ornate flowers (see Letter by H-F. Hsu et al.). As extensively investigated in Arabidopsis, the overlapping domains of expression of different MADS-box transcription factors define the architecture of a flower. In orchids further layers of complexity have been added with additional MADS-box proteins creating the lip structure characteristic of orchid flowers and which serves as a landing platform for pollinating insects. A further non-model feature of orchids is their naked seeds lacking endosperm to supply seedlings with energy following germination. Instead an orchid seed must initially develop parasitically on a fungus; some orchid species never grow leaves and continue as parasites for their full life span. The naked seeds of orchids seem perfectly adapted to wind dispersal, although a Brief Communication from $\mathrm{K}$. Suetsugu et al. shows that at least one species has the help of birds.

Despite their unique qualities, dandelions and orchids remain relatively conventional. Where should we look for a truly non-model plant? What is the plant equivalent to the duckbilled platypus, the venomous, egg laying mammal so atypical that European zoologists thought it a hoax when the first specimens were sent back from Australia? The venus fly trap (Dionaea muscipula) might make a claim for its fast-acting trap to capture and digest insect prey. Or yareta (Azorella compacta; pictured), which look like green boulders and have been slowly growing in the extreme conditions of the high Andes for 3,000 years or more.

Such extreme organisms are also intensively studied. Dionaea is under scrutiny for, among other things, its electrically excitable cells that have arisen independently of the nerve cells of animals and control its trap. Yareta has a unique secondary metabolism producing a range of diterpenoids that have medicinal potential.

All plants, indeed all organisms, are both atypical and representative at the same time. Each followed its individual evolutionary route from common ancestor to extant individual. It is thus important to remember that model plants such as Arabidopsis, Brachipodioum or Marchantia do not represent the Platonic ideal of a plant. They simply have characteristics that make them uniquely easy for us to study. 